\title{
PERIODICITY AND DECOMPOSABILITY \\ OF BASIN BOUNDARIES \\ WITH IRRATIONAL MAPS ON PRIME ENDS
}

\author{
RUSSELL B. WALKER
}

\begin{abstract}
Planar basin boundaries of iterated homeomorphisms induce homeomorphisms on prime ends. When the basin is connected, simply connected, and has a compact connected boundary, the space of prime ends is a topological circle. If the induced homeomorphism on prime ends has rational rotation number, the basin boundary contains periodic orbits. Several questions as to basin boundary periodics, decomposability, and minimality, when the induced map on prime ends has irrational rotation number, are answered by construction of both homeomorphisms and diffeomorphisms. Examples in the literature of basin boundaries with interesting prime end dynamics have been sparse. Prime end dynamics has drawn recent interest as a natural tool for the study of strange attractors.
\end{abstract}

In their 1951 manuscript entitled Some fixed point theorems, M. L. Cartwright and J. E. Littlewood proved that if the induced map on Carathéodory's prime ends has rational rotation number, then a connected basin boundary of an orientation preserving planar homeomorphism must contain a periodic orbit [CL1, C].

In this note we explore the relationship between aperiodic maps induced on the circle of prime ends and the periodicity, minimality, and decomposability of basin boundaries. Attractors (as frequently defined) serve as connected basin boundaries and one usually requires them to contain dense orbits. The existence of a dense orbit would distinguish an attractor from a union of periodic orbits or other attracting sets. Can the map on prime ends, induced from the basin itself, help detect these dense orbits? We construct a basin separating attractor (of a $C^{1}$ diffeomorphism) containing no dense orbits which induces pure irrational rotation of both circles of prime ends (Example A). But some minimal attractors (all orbits dense) can induce two Denjoy maps on prime ends (on both sides) which have no dense orbits [Den] (Example E).

Conceptually one imagines the map on the circle of prime ends as the action of the planar homeomorphism on the basin boundary as viewed from the basin. Precise definitions of key terminology follow. An excellent reference is $J$. N. Mather's exposition [M].

Received by the editors October 28, 1988 and, in revised form, March 14, 1989.

1980 Mathematics Subject Classification (1985 Revision). Primary 54H20; Secondary 58F12, $58 \mathrm{~F} 22$. 
Recently, K. Alligood, T. Sauer, and J. Yorke revived the study of prime end dynamics [AS, AY]. They suggest that computer-generated pseudo orbits, near attractors, may be best interpreted in the context of the space of prime ends. In other recent work, M. Barge and R. Gillette prove that under certain conditions a "cobasin boundary," which induces two rational rotation numbers on prime ends with different denominators (in reduced form), must be indecomposable [BG]. Their work is a step towards understanding the relationship between the dynamics of maps on prime ends and the topology of basin boundaries. They resolve a question due to Cartwright and Littlewood concerning the nature of the van der Pol oscillator [CL2, L].

To our knowledge, two examples exist in the literature of planar homeomorphisms which induce at a basin boundary a homeomorphism on prime ends with irrational rotation number; one has an imbedded circle basin boundary, the other has M. Handel's hereditarily indecomposable pseudo circle [H]. Once more, the purpose of this manuscript is to answer several natural questions concerning induced homeomorphisms on circles of prime ends which have irrational rotation numbers. To simplify their precise statements, when a basin boundary separates two basins and is the boundary of each, we will refer to it as a cobasin boundary. We answer by example these questions: If the homeomorphism on prime ends is conjugate to pure irrational rotation (i.e., is minimal), must the action of the planar homeomorphism on the basin boundary be transitive, that is, must it contain a dense orbit? We ask the analogous question about cobasin boundaries. If the homeomorphism on the circle of prime ends has irrational rotation number can a basin boundary contain periodic orbits? If a cobasin boundary induces two conjugate pure irrational rotations as maps on prime ends must the boundary be either a circle or a pseudo circle?

G. D. Birkhoff constructed a cobasin boundary in an annular twist map which induced two nonconjugate homeomorphisms on prime ends [Bi]. The cobasin boundary was indecomposable; it could not be decomposed into the union of two proper compact connected subsets. Recently M. Barge and R. Gillette proved that if a cobasin boundary contains two periodic orbits with relatively prime periods but no fixed points, it must be indecomposable [BG]. It is natural to ask, must cobasin boundaries which induce nonconjugate prime end maps be indecomposable or contain indecomposable compact connected subsets?

These first questions concern what dynamics on the basin boundaries are forced by certain dynamics on the circle of prime ends. Reversing the logic, must minimal cobasin boundaries induce minimal homeomorphisms on prime ends?

We now list five examples which answer each of these questions in the negative. Included is a theorem concerning allowable pairs of induced circle homeomorphisms on prime ends for basin separating boundaries (which need not be cobasin boundaries). We make two conjectures. 
Example A. We construct a $C^{1}$ diffeomorphism with a basin separating boundary, $\Lambda$, containing no dense orbits which induces conjugate irrational rotations on the two circles of prime ends. This example may be "wedged open" producing a cobasin boundary containing no dense orbits which induces two maps on circles of prime ends, one pure irrational rotation, the other Denjoy having the same irrational rotation number [Den]. In both cases the boundary is hereditarily decomposable.

Example B. There exists a $C^{1}$ planar diffeomorphism with a basin separating boundary containing periodic orbits which has a Denjoy induced map on prime ends.

Theorem. Let $f_{1}$ and $f_{2}$ be two circle homeomorphisms. Assume there do not exist $\alpha$ and $\beta$ such that $\alpha / 2 \pi$ and $\beta / 2 \pi$ are irrational, $\alpha \neq \beta$, with $f_{1}$ conjugate to rotation by $\alpha$, and $f_{2}$ conjugate to rotation by $\beta$. Then there exists a $C^{1}$ planar diffeomorphism with a plane separating basin boundary having (up to topological conjugacy) these two, as induced maps on prime ends.

Example C. There exists a planar homeomorphism with an hereditarily decomposable circularly chainable cobasin boundary which induces two minimal homeomorphisms on prime ends, but which is not a circle.

Example D. Next, we construct a $C^{1}$ planar diffeomorphism with a transitive cobasin boundary (dense orbit) which induces two nontransitive Denjoy homeomorphisms on the circles of prime ends.

Example E. Relying on the techniques exhibited in Examples $C$ and D we construct a planar homeomorphism with a minimal cobasin boundary (every orbit dense) which induces two Denjoy homeomorphisms on the circles of prime ends (no dense orbits).

Conjecture 1. A cobasin boundary is minimal under the action of a planar homeomorphism if it induces two conjugate irrational rotations on the two circles of prime ends.

Conjecture 2. No cobasin boundary induces two nonconjugate irrational rotations on prime ends.

\section{TeRminology}

Let $F$ be a planar homeomorphism. For $p \in \mathbf{R}^{2},\left\{F^{n}(p) \mid n \in \mathbf{Z}\right\}$ is the orbit of $p$ under $F$. When $F^{n}(p)=p$ for some $n, p$ is periodic. If there exists $\left\{n_{1}, n_{2}, \ldots\right\}$ such that $F^{n_{k}}(p) \rightarrow p$ as $k \rightarrow \infty$, the orbit or the point is recurrent. $p$ wanders if there exists an open neighborhood $U$ such that $F^{n}(U) \cap U=\varnothing$ for all $n \neq 0$. The set $\Lambda \subset \mathbf{R}^{2}$ is invariant if $F(\Lambda)=\Lambda .\left.F\right|_{\Lambda}$ or $\Lambda$ is transitive if an orbit of $F$ is dense in $\Lambda$. If $\Lambda$ is compact and all orbits contained in $\Lambda$ are dense in $\Lambda, \Lambda$ is minimal. Two homeomorphisms $F$ and $G$ are conjugate if there exists a third homeomorphism $h$ so that $F \circ h=h \circ G$. 
An open set $U$ is called a basin of attraction if there exists an open subset $V \subsetneq U$ such that $F(V) \subsetneq V$ and $U=\bigcup_{n<0} F^{n}(V) . U$ is called a negative basin of attraction if it is a basin of attraction of $F^{-1}$. We use just basin when the distinction is not relevant. If $\Lambda$ is closed and connected and there exists an open set $\Lambda \subset W \subset \mathbf{R}^{2}$ such that $W \backslash \Lambda=U_{1} \cup U_{2}$, where $U_{1}$ and $U_{2}$ are connected basins, $U_{1} \cap U_{2}=\varnothing$, and $\Lambda=\partial U_{1}=\partial U_{2}$, then $\Lambda$ will be called a cobasin boundary. Throughout, basin boundaries will be compact and connected.

Let $\Omega(F)$ denote the points of $F$ which do not wander or are nonwandering. We call a circle homeomorphism $F$ Denjoy-like if $F$ has no periodic points and $\Omega(F) \subset S^{1}$ is a Cantor set [Dev]. Let $\widetilde{F}$ be a lift of $F$ to the universal covering space $\mathbf{R} . \rho(F)$ is the rotation number of $F$, where

$$
\rho(F)=\left[(1 / n) \lim _{n \rightarrow \infty} \widetilde{F}^{n}(0)\right] \quad(\bmod 1) .
$$

This well-defined real number is independent of the choice of lift. All Denjoylike circle homeomorphisms have irrational rotation numbers.

A compact connected metric space is called a continuum. One is decomposable if it can be written as the union of two proper subcontinua; otherwise, it is indecomposable. (For example, on a compact 2-manifold, the closure of the unstable manifold of a hyperbolic saddle point which contains a transverse homoclinic point is an indecomposable continuum.) If every subcontinuum is decomposable it is called hereditarily decomposable. If every subcontinuum is indecomposable, it is hereditarily indecomposable (for example, the pseudo circle). A continuum $\Lambda$ is circularly chainable if for every $\varepsilon>0$, there is a projection of $\Lambda$ onto the circle such that all point-inverses have diameter less than $\varepsilon$.

Prime ends. We now define the prime ends of basin boundaries, $\Lambda=\partial U \subset \mathbf{R}^{2}$. Our notation follows that of J. Mather [M]. Let $\left\{V_{1} \supset V_{2} \supset \cdots\right\}$ be a chain of open, connected subsets of $U$ such that, (a) $\partial V_{i} \neq \varnothing$ and is connected, for each $i$, and (b) $\mathrm{Cl}\left(\partial V_{i} \cap U\right) \cap \mathrm{Cl}\left(\partial V_{j} \cap U\right)=\varnothing$, for $i \neq j$. Assume two chains $\sigma=\left\{V_{1} \supset V_{2} \supset \cdots\right\}$ and $\tau=\left\{W_{1} \supset W_{2} \supset \cdots\right\}$ are given. Then $\tau$ divides $\sigma$ if for every $i$ there is a $j$ such that $W_{j} \subset V_{i}$. Two chains are equivalent if each divides the other. A chain is prime if any chain which divides it is equivalent to it. A prime point is the equivalence class of a prime chain.

If $\left\{D_{1} \supset D_{2} \supset \cdots\right\}$ is a family of closed disks in $U$ such that for all $i$, $D_{i+1} \subset$ int $D_{i}$, and $x \in D_{i}$, then int $D_{1} \supset$ int $\left.D_{2} \supset \cdots\right\}$ is a prime chain. Let $\omega(x)$ denote the prime point it represents. A prime end is a prime point which is not of the form $\omega(x)$ for any $x \in U$. If $e=\left\{V_{1} \supset V_{2} \supset \cdots\right\}$ represents a prime end, then $\partial V_{i} \cap U$ is called a crosscut. The intersection $\mathscr{I}(e)=\bigcap_{i} \mathrm{Cl} V_{i}$ is called the impression of $e$. In the case of M. Handel's imbedded pseudo circle, all impressions are the whole pseudo circle. On the other hand, for an imbedded circle, all impressions are single points; their prime ends are then called trivial. 
We now define a topology on $\widehat{U}$, the set of prime points of $U$. Let $W$ be open in $U$. Let $e \in \hat{U}$ and assume $\left\{V_{1} \supset V_{2} \supset \cdots\right\}$ represents $e$. Then $e$ divides $W$ if there exists $i$ such that $V_{i} \subset W$. Let $\widetilde{W}$ denote the set of all prime points which divide $W$. Then $\{\widetilde{W} \mid W$ is open in $U\}$ forms a basis for our topology on $\widehat{U}$.

Let $F$ be a planar homeomorphism which leaves $U$ invariant. Then $F$ preserves division and "primeness" of chains; if $\left\{V_{1} \supset V_{2} \supset \cdots\right\}$ represents $e \in \widehat{U}$ and $e$ divides $W$, then the chain $\left\{F\left(V_{1}\right) \supset F\left(V_{2}\right) \supset \cdots\right\}$ represents a prime point which divides $F(W)$. Denote this prime point by $\widehat{F}(e)$. The map $\widehat{F}: \widehat{U} \rightarrow \widehat{U}$ is a homeomorphism onto its image.

All of our basin boundaries, $\partial U$, are continua and contain more than one point. It was known to Caratheodory that the collection of prime ends of $U$, denoted by $\partial \widehat{U}$, is homeomorphic to a circle; furthermore, $\widehat{F} \mid \partial \widehat{U}$ is a circle homeomorphism. We refer to $\widehat{F} \mid \partial \hat{U}$ as the induced homeomorphism on the circle of prime ends for $\Lambda=\partial U$. A prime end $e$ wanders under $\widehat{F}$ if for every chain $\left\{V_{1} \supset V_{2} \supset \cdots\right\}$ representing $e$, there exists $i_{0}$ such that $\widehat{F}^{n}(e)$ does not divide $V_{i_{0}}$ for $n \neq 0$. Since $\widehat{F} \mid \partial \widehat{U}$ is a circle homeomorphism, $e \in \partial \widehat{U}$ is recurrent if it is nonwandering.

\section{Proof of The theorem and Example B}

To familiarize the reader with the various concepts and terminology used, we start with the proof of the theorem. Example B follows immediately. We proceed to the construction of Examples $C$ and $D$ which introduce the reader to some of the techniques used in the construction of Examples A and E. Several of the arguments make use of various well-known lemmas from the theory of circle homeomorphisms (see for example Z. Nitecki [Ni], and R. Devaney [Dev]).

Proof of the theorem. Let $f_{1}=f_{2}=$ rotation by an irrational on $S^{1}$. Let $F(r, \theta)=\left(\sqrt{r}, f_{1}(\theta)\right)$. Then $\Lambda=\{r=1\}$ separates the two negative basins of attraction $\{r>1\}$ for $(0,0)$ and $\{r<1\}$ for $\infty . F_{0}$ induces (maps conjugate to) $f_{1}$ on both circles of prime ends. Smooth $F$ near $(0,0)$ without disturbing these properties.

Next assume $f_{1}$ is any circle homeomorphism and that $f_{2}$ is Denjoy-like. Because every circle homeomorphism has a $C^{1}$ conjugate, we assume $f_{1}$ and $f_{2}$ are diffeomorphisms. First, let $F_{0}=\left(g(r), f_{r}(\theta)\right)$ be a planar diffeomorphism for which $g(r)=\sqrt{r}$, except near $(0,0)$, and $f_{r}(\theta)$ is a $C^{1}$ circle isotopy such that each $f_{r}$ is a $C^{1}$ diffeomorphism for all $r$ and

$$
f_{r}(\theta)= \begin{cases}f_{1}(\theta), & r \leq 1, \\ f_{2}(\theta), & r \geq 2 .\end{cases}
$$

Let $B=\left\{(r, \theta) \mid \sqrt{2} \leq r \leq 2\right.$ and $\left.\theta \in \Omega\left(f_{2}\right)\right\}$, and $\Lambda=\left[\bigcup_{n \geq 0} F_{0}^{n}(B)\right] \cup\{r=1\}$ (see Figure 1). Now smoothly adjust $F_{0}$ near $B$ forming the diffeomorphism $F$ such that $F(\Lambda)=\Lambda$ and $\mathbf{R}^{2} \backslash \Lambda$ is the union of two negative basins of 


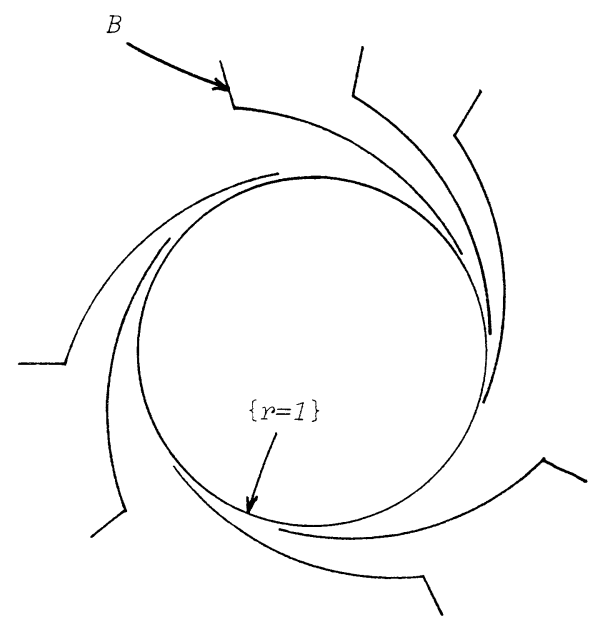

Figure 1

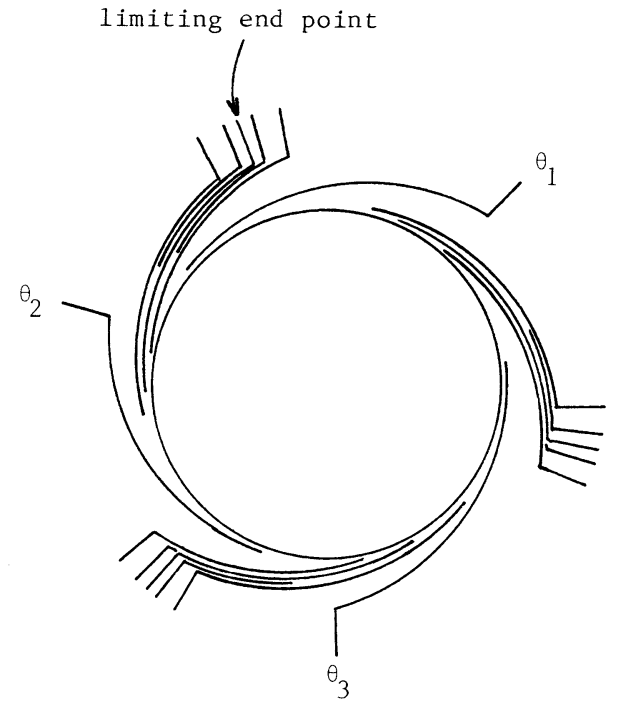

Figure 2

attraction, $U_{1}$ for $(0,0)$ and $U_{2}$ for $\infty$. Further require that points on the "hairs of $\Lambda, " \Lambda \backslash\{r=1\}$, except those at the "endpoints," $\Lambda \cap\{r=2\}$, continue to drift towards $\{r=1\}$ under $F$.

$F$ induces $f_{1}$ on the circle of prime ends corresponding to $U_{1}$. The endpoints of $\Lambda$ are the impressions of nonwandering trivial prime ends of $U_{2}$. These prime ends are mapped about the circle of prime ends by $f_{2}$. All other prime ends of $U_{2}$ wander. Since for Denjoy-like homeomorphisms, the map on the nonwandering set determines its conjugacy class, the induced map on prime ends, corresponding to $U_{2}$, is $f_{2}$ (or its conjugacy class).

Now assume $f_{1}$ is any $C^{1}$ circle diffeomorphism, that $\rho\left(f_{2}\right) \in \mathbf{Q}$, but $\Omega\left(f_{2}\right) \neq S^{1}$ (see Figure 2). Let $F_{0}=\left(g(r), f_{r}(\theta)\right)$ be as in the previous case. $S^{1} \backslash \Omega\left(f_{2}\right)$ consists of a countable union of open intervals, $\bigcup_{i \in \mathbf{N}} I_{i}$. Choose $\theta_{i} \in I_{i}$, each $i$, and let $A=\left\{f_{2}^{m}\left(\theta_{i}\right) \mid i \in \mathbf{N}\right.$ and $\left.m \in \mathbf{Z}\right\}$. Let $B=\left\{(r, \theta) \mid \sqrt{2} \leq r \leq 2, \theta \in A\right.$ or $\left.\theta \in \Omega\left(f_{2}\right)\right\}$. Again,

$$
\Lambda=\left[\bigcup_{n \geq 0} F_{0}^{n}(B)\right] \cup\{r=1\} .
$$

Adjust $F_{0}$ near $B$, forming $F$, as in the previous case. The "limiting endpoints" $A_{0}=\left\{(r, \theta) \mid r=2\right.$ and $\left.\theta \in \Omega\left(f_{2}\right)\right\}$ are nonwandering and shuttled around $\{r=2\}$ by id $\times f_{2}$. All other points of $\Lambda \backslash\{r=1\}$ wander. Let $p \in A_{0}$ and assume $p$ is fixed by id $\times f_{2}^{m}$, for some $m$. Let $q \in \Lambda \cap\{r=2\}$ have the property that $f_{2}^{m l}(q) \rightarrow p$ as $l \rightarrow \infty$. If $\left\{V_{1} \supset V_{2} \supset \cdots\right\}$ represents a prime end with impression containing $p$, then any prime end with impression along the hair of $\Lambda$ through $q$, eventually under $\widehat{F}^{m}$, divides $V_{j}, j \geq 1$. 
The map $\psi$ that carries $e \in \partial \widehat{U}_{2}$ having impression containing $(2, \theta) \in A_{0}$ to $\theta$ is a homeomorphism from $\Omega\left(\left.\widehat{F}\right|_{\partial \widehat{U}_{2}}\right)$ to $\Omega\left(f_{2}\right)$. Furthermore $\psi$ conjugates $\left.\widehat{F}\right|_{\partial \widehat{U}_{2}}$ and $f_{2}$ on these nonwandering sets. Since the wandering points of $\widehat{F} \mid \partial \widehat{U}_{2}$ and $f_{2}$ wander in the same "sense" across open intervals, $\psi$ extends to a conjugacy between $\left.\widehat{F}\right|_{\partial \widehat{U}_{2}}$ and $f_{2}$.

In the last case, when $f_{1}$ is any $C^{1}$ circle diffeomorphism with $\rho\left(f_{2}\right) \in \mathbf{Q}$ and $\Omega\left(f_{2}\right)=S^{1}$, let $B=\{(r, \theta) \mid \sqrt{2} \leq r \leq 2$ and $\theta=0\}$. Let $\Lambda$ and $F$ be as above. Now all prime ends are periodic; $\left.\widehat{F}\right|_{\partial \widehat{U}_{2}}$ is conjugate to $f_{2}$.

Example B. In the proof of the theorem, let $f_{1}$ have rational rotation number and let $f_{2}$ be a Denjoy-like homeomorphism. The basin separating invariant set is depicted by Figure 1 .

\section{EXAMPLE C}

We form the cobasin boundary $\Lambda$ by radially adding together infinitely many irrationally rotated and radially rescaled copies of the Warsaw circle $\Lambda_{0}[\mathrm{~K}]$ (see Figure 3).

W. H. Gottschalk and G. A. Hedlund [GH] constructed the same continuum which supports a minimal homeomorphism. We now take care to extend a similar homeomorphism on $\Lambda$ to the plane.

To ease the radial addition computations, we make the construction on $\mathbf{R} \times S^{1}$ in $(r, \theta)$-coordinates. Let

$$
f_{ \pm}(\theta)= \begin{cases}\sin (1 / \theta), & 0<|\theta| \leq 1 / \pi, \\ 0, & |\theta|>1 / \pi, \theta \in[-\pi, \pi], \\ \pm 1, & \theta=0 .\end{cases}
$$

Then $\Lambda_{0}=\left\{(r, \theta) \mid-\pi \leq \theta \leq \pi, f_{-}(\theta) \leq r \leq f_{+}(\theta)\right\} . \Lambda_{0}$ separates the cylinder into two components: $U_{0}^{+}$contains the large radial values, $U_{0}^{-}$the small. Next let $g: \mathbf{R} \times S^{1} \rightarrow \mathbf{R}$ be a smooth bounded function such that $g>0$

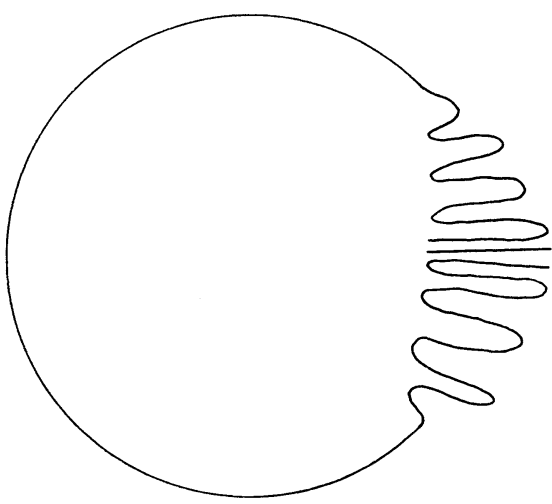

FIGURE 3. $\Lambda_{0}$ 
on $U_{0}^{+}, g<0$ on $U_{0}^{-}$, and $g^{-1}(0)=\Lambda_{0}$. Let $Y$ be the vectorfield on $\mathbf{R} \times S^{1}$ given by $Y(p)=g(p) \frac{\partial}{\partial r}$. Let $\psi_{t}$ be the flow generated by $Y$.

Denote by $R\left(\theta_{0}\right)=\left\{\theta=\theta_{0}\right\} \subset \mathbf{R} \times S^{1}$. The smooth map $b: \mathbf{R} \times S^{1} \rightarrow \mathbf{R}$ strictly increases along $R(\theta) \cap\left[U_{0}^{+} \cup U_{0}^{-}\right]$and $b^{-1}(0)=\Lambda_{0}$ :

$$
b(p)= \begin{cases}e^{-t} & \text { when } \psi_{t}(p)=2, \\ 0 & \text { when } p \in \Lambda_{0}, \\ e^{t} & \text { when } \psi_{t}(p)=-2 .\end{cases}
$$

The smooth map $h: \mathbf{R} \times S^{1} \supset$ give by $h(p)=\left(b(p), \pi_{\theta}(p)\right) \quad\left(\pi_{\theta}=\right.$ projection onto $S^{1}$ ) collapses $\Lambda_{0}$ onto the circle $\{r=0\}$ while preserving $\theta$-coordinates. $h$ is a smooth diffeomorphism, off the "limit bar" $h^{-1}(0,0)=\{(r, 0)|| r \mid \leq 1\} \subset$ $\Lambda_{0}$, onto $\mathbf{R} \times S^{1} \backslash\{(0,0)\}$. For later use note that

$$
h^{-1}(\alpha, \theta)= \begin{cases}\psi_{\ln \alpha}(2, \theta), & \alpha>0, \\ R(\theta) \cap \Lambda_{0}, & \alpha=0, \\ \psi_{\ln |\alpha|}(-2, \theta), & \alpha<0 .\end{cases}
$$

Now fix $\omega \in \mathbf{R}$ such that $\omega / 2 \pi \neq \mathbf{Q}$. For each $\alpha \in \mathbf{R}$, let

$$
l(\alpha)=\left\{(r, \theta) \mid r \in \sum_{n=-\infty}^{\infty} \frac{\pi_{r} h^{-1}(\alpha, \theta-n \omega)}{2^{|n|}}\right\} .
$$

Then $\Lambda=l(0)$ (see Figure 4).

We leave to the reader to verify the following five technical lemmas which have straightforward proofs.

Lemma 1. When $\alpha \neq 0, l(\alpha)$ is an essential circle on $\mathbf{R} \times S^{1}$.

Lemma 2. $l(\alpha)$ is compact and connected for all $\alpha$.

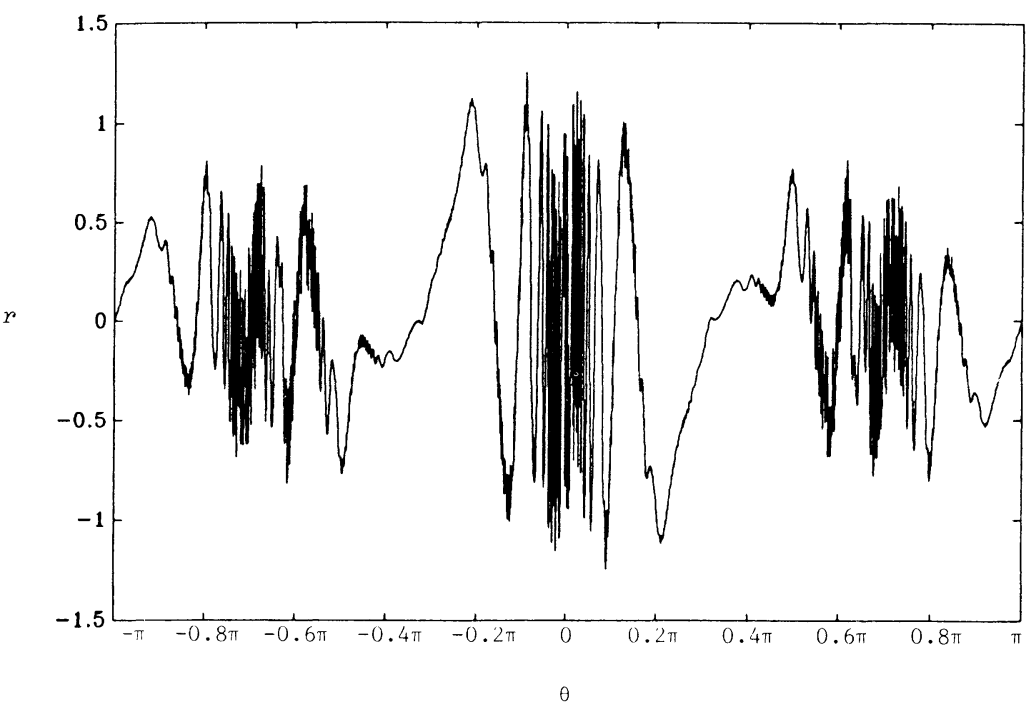

FIGURE 4 
Lemma 3. If $\alpha<\beta$ then $l(\alpha) \cap l(\beta)=\varnothing$ and $\pi_{r}[R(\theta) \cap l(\alpha)]<\pi_{r}[R(\theta) \cap l(\beta)]$ for all $\theta$.

Lemma 4. $l(a)=\partial \bigcup_{\alpha \geq a} l(\alpha)$.

Lemma 5. If $c<d$ and neither is 0 , then $A(c, d)=\bigcup_{\alpha \in(c, d)} l(\alpha)$ is an open annular neighborhood of $l_{\gamma}$, when $\gamma \in(c, d)$.

We now prepare to define the desired cylinder homeomorphism $F$. Let $\alpha(p)$ be such that $p \in l[\alpha(p)]$. By Lemma 3, $\alpha$ is well defined.

Lemma 6. $\alpha: \mathbf{R} \times S^{1} \rightarrow \mathbf{R}$ is continuous.

Proof. By Lemma 5, given $\left\{p_{n} \rightarrow p_{0}\right\} \subset \mathbf{R} \times S^{1}$ and $\varepsilon>0$, there exists $N$ such that for all $n>N, p_{n} \in A\left(\alpha\left(p_{0}\right)-\varepsilon, \alpha\left(p_{0}\right)+\varepsilon\right)$. (If in fact $\alpha\left(p_{0}\right) \pm \varepsilon=0$, use $\alpha\left(p_{0}\right) \pm \varepsilon / 2$ instead.) Equivalently, for all $n>N, p_{n} \in l(\alpha)$ for some $\alpha\left(p_{0}\right)-\varepsilon<\alpha<\alpha\left(p_{0}\right)+\varepsilon$.

The next collection of maps, $\left\{s_{i}: \mathbf{R} \times S^{1} \rightarrow \mathbf{R}\right\}$, sum to the radial coordinate:

$$
s_{i}(p)= \begin{cases}\frac{\pi_{r} h^{-1}\left(\alpha(p), \pi_{\theta}(p)-i \omega\right)}{2^{|i|}}, & |\alpha(p)|>0, \\ \frac{\pi_{r} h^{-1}\left(0, \pi_{\theta}(p)-i \omega\right)}{2^{|i|}}, & \alpha(p)=0 \text { and } \pi_{\theta}(p) \neq i \omega, \\ \pi_{r}(p)-\sum_{j \neq i} s_{j}(p), & \alpha(p)=0 \text { and } \pi_{\theta}(p)=i \omega .\end{cases}
$$

Because $p \in l(\alpha(p)), \sum_{-\infty}^{\infty} s_{i}(p)=\pi_{r}(p)$. We will use that $s_{i}(p)=\pi_{r}(p)-$ $\sum_{j \neq i} s_{j}(p)$ for all $p$.

Lemma 7. $s_{i}$ is continuous for all $i$.

Proof. That $\left[\pi_{r}(q)-\sum_{j \neq i} s_{j}(q)\right] \rightarrow\left[\pi_{r}(p)-\sum_{j \neq i} s_{j}(p)\right]$ as $q \rightarrow p$, when $\alpha(p)=$ 0 and $\pi_{\theta}(p)=i \omega$, follows from the fact that the tails $\sum_{|i|>N}\left|s_{i}(p)\right| \rightarrow 0$ as $N \rightarrow \infty$.

Let $F: \mathbf{R} \times S^{1}$ be given by

$$
F(p)=\left(2 \sum_{-\infty}^{-1} s_{i}(p)+\frac{1}{2} \sum_{0}^{\infty} s_{i}(p), \pi_{\theta}(p)+\omega\right) .
$$

It follows from Lemma 7 that $F$ is continuous.

Lemma 8. $\Lambda$ is the boundary of both

$$
U^{+}=\{q \mid \alpha(q)>0\} \text { and } U^{-}=\{q \mid \alpha(q)>0\} .
$$

Proof. Briefly, near each $p \in \Lambda$, there exists $q_{0} \in \Lambda$ such that $\pi_{0}\left(q_{0}\right) \neq n \omega$ for any $n \in \mathbf{Z}$. In turn, near $q_{0}$ there exist points on the same ray as $q_{0}$ within $U^{+}$, and also within $U^{-}$.

The key to showing that $F$ is injective is the following: 
Lemma 9. $F(\Lambda)=\Lambda$.

Proof. Let $p \in \Lambda$. If $\pi_{\theta}(p) \neq i \omega$ for $i \geq 0$,

$$
\frac{1}{2} s_{i}(p)=\frac{h^{-1}\left(0, \pi_{\theta}(p)+\omega-(i+1) \omega\right)}{2^{i+1}}=s_{i+1}\left(\Lambda \cap R\left(\pi_{\theta}(p)+\omega\right)\right) .
$$

When $i<0,2 s_{i}(p)=s_{i+1}\left(\Lambda \cap R\left(\pi_{\theta}(p)+\omega\right)\right)$. Since $\sum s_{i}(q)=\pi_{r}(q), \pi_{r} F(p)=$ $\pi_{r}\left(\Lambda \cap R\left(\pi_{\theta}(p)+\omega\right)\right)$. Thus $F(p)=\Lambda \cap R\left(\pi_{\theta}(p)+\omega\right)$.

When $\pi_{\theta}(p)=i_{0} \omega$ and $i_{0}>0$,

$$
p \in \Lambda \Leftrightarrow\left|\pi_{r}(p)-\sum_{j \neq i_{0}} \frac{f_{+}(\theta-j \omega)}{2^{|j|}}\right| \leq \frac{1}{2^{i_{0}+1}} .
$$

But for $j \neq i_{0}$,

$$
s_{j}(p)=\frac{\pi_{r} h^{-1}(0, \theta-j \omega)}{2^{|j|}}=\frac{f_{+}(\theta-j \omega)}{2^{|j|}} .
$$

So

$$
p \in \Lambda \Leftrightarrow\left|\pi_{r}(p)-\sum_{j \neq i_{0}} s_{j}(p)\right| \leq \frac{1}{2^{i_{0}+1}} \Leftrightarrow\left|s_{i_{0}}(p)\right|<\frac{1}{2^{i_{0}+2}} .
$$

Thus

$$
\left|\pi_{r} F(p)-2 \sum_{-\infty}^{-1} s_{j}(p)-\frac{1}{2} \sum_{0}^{\infty} s_{j}(p)\right| \leq \frac{1}{2^{i_{0}}}
$$

or $F(p) \in \Lambda \cap R\left(\pi_{\theta}(p)+\omega\right)$. Similarly for $i_{0}<0$ and for $F^{-1}$.

Lemma 10. $F$ is injective.

Proof. We do the case $p, q \in \Lambda, p \neq q$, and $\pi_{\theta}(p)=\pi_{\theta}(q)$. Then $\pi_{\theta}(p)=$ $i_{0} \omega$ for some $i_{0} \in \mathbf{Z}$. But for $i \neq i_{0}, \pi_{r} h^{-1}\left(0, \pi_{\theta}(p)-i \omega\right)$ is single valued in $p ; s_{i}(p)=s_{i}(q)$ when $i \neq i_{0}$. Thus

$$
\begin{aligned}
\pi_{r} F(p)-\pi_{r} F(q) & =\left\{\begin{array}{l}
2\left[s_{i_{0}}(p)-s_{i_{0}}(q)\right] ; i_{0} \leq-1 \\
\frac{1}{2}\left[s_{i_{0}}(p)-s_{i_{0}}(q)\right] ; i_{0}>0
\end{array}\right\} \\
& =\left\{\begin{array}{l}
2\left[\pi_{r}(p)-\pi_{r}(q)\right] ; i_{0}<0 \\
\frac{1}{2}\left[\pi_{r}(p)-\pi_{r}(q)\right] ; i_{0} \geq 0
\end{array}\right\} .
\end{aligned}
$$

But since $\pi_{\theta}(p)=\pi_{\theta}(q)$ and $p \neq q, \pi_{r}(p) \neq \pi_{r}(q)$.

The inverse $F^{-1}(p)=\left(2 \sum_{-\infty}^{0} s_{i}(p)+\frac{1}{2} \sum_{1}^{\infty} s_{i}(p), \theta-\omega\right)$ is continuous by Lemma 7.

$\Lambda$ can be projected with small diameter point inverses onto a similar radial sum of "near Warsaw circles" which are true circles. These circles are identical to Warsaw circles except near the limit bar they "jump across." A precise argument along these lines concludes that $\Lambda$ is circularly chainable. 
Lemma 11. $\Lambda$ is the cobasin boundary between $U^{+}=\{q \mid \alpha(q)>0\}$ and $U^{-}=$ $\{q \mid \alpha(q)<0\}$ under a homeomorphism near $F$.

Proof. Using the definition of $\left\{s_{i}(p)\right\}$ one checks that $\alpha(F(p))=\alpha(p)$. But each leaf $l(\alpha)$ intersects each ray in a unique point (off $\Lambda)$. Thus $F(l(\alpha))=$ $l(\alpha)$. Let $g$ be a homeomorphism near to the identity which fixes $\Lambda$, preserves radial coordinates, and has the property that $g(l(\alpha))=l(\beta)$ for $|\alpha| \leq|\beta|$. Then $g \circ F$ is the desired homeomorphism.

Lemma 12. $\Lambda$ is hereditarily decomposable.

Proof. Let $H$ be a subcontinuum of $\Lambda$. Then either $\pi_{\theta} H=$ a point, $\pi_{\theta} H=$ $[\alpha, \beta]$, where $|\alpha-\beta|<2 \pi$, or $\pi_{\theta} H=S^{1}$. In the first case $H$ is a subset of an arc. In the second, let $\alpha<\gamma<\beta$ and consider $H_{1}=\pi_{\theta}^{-1}[\alpha, \gamma]$ and $H_{2}=\pi_{\theta}^{-1}[\gamma, \beta]$. Then $H, H_{1}$, and $H_{2}$ are proper subcontinua of the cobasin $\Lambda$. By Cartwright and Littlewood [CL1], none may be plane separating. By $\mathrm{M}$. Newman [Ne], $H \cap H_{1}$ and $H \cap H_{2}$ are continua because $H \cup H_{1}$ and $H \cup H_{2}$ are not plane separating. But $H \subset \pi_{\theta}^{-1}[\alpha, \beta]$, so $\left[H \cap H_{1}\right] \cup\left[H \cap H_{2}\right]$ is a decomposition of $H$. A similar argument works when $\pi_{\theta} H=S^{1}$.

\section{EXAMPLES D AND E}

Example D. A. S. Besicovitch [Be1, Be2] constructed a planar homeomorphism with a dense orbit which is irrational rotation on rays. Fix $\omega / 2 \pi \neq \mathbf{Q}$. Let $A=[0,1] \times S^{1}$. First adapt the Besicovitch map forming a homeomorphism $f: \mathbf{R} \times S^{1} \supset$, where $f(r, \theta)=\left(f_{r}(r, \theta), \theta+\omega\right)$ has the following properties: (i) $f_{r}(r, \theta)=r$ for $(r, \theta) \notin A$, and (ii) there exists $p_{0}$ and $q_{0}$ with dense orbits in $A$ (under $f$ ) such that $\left\{\pi_{\theta} p_{0}+n \omega\right\} \cap\left\{\pi_{\theta} q_{0}+m \omega\right\}=\varnothing$ for all $n, m \in \mathbf{Z}$.

Denote $\theta_{n}(p)=\pi_{\theta}(p)+n \omega \in S^{1}$ and again $R\left(\theta_{0}\right)=\left\{\theta=\theta_{0}\right\} \subset \mathbf{R} \times S^{1}$. Let $A^{+}=\{r>1\}$ and $A^{-}=\{r<0\}$. Slice open $\mathbf{R} \times S^{1}$ and replace each $R\left(\theta_{n}\left(p_{0}\right)\right)$ by $P_{n}$ and each $R\left(\theta_{n}\left(q_{0}\right)\right)$ by $Q_{n}$. These two infinite wedges (see Figure 5a) have the following properties:

(i) $\partial P_{n}$ and $\partial Q_{n}$ are piecewise linear.

(ii) There exists $\left\{a_{n}\right\} \subset\{r=1\}$ and $\left\{d_{n}\right\} \subset\{r=0\}$ such that

$$
\partial P_{n} \cap A^{+}=(1, \infty) \times\left\{\pi_{\theta} a_{n}\right\}
$$

and

$$
\partial Q_{n} \cap A^{-}=(-\infty, 0) \times\left\{\pi_{\theta} d_{n}\right\} .
$$

(iii) There exists $\left\{b_{n}\right\},\left\{c_{n}\right\} \subset\{r=0\}$ and $\left\{e_{n}\right\},\left\{k_{n}\right\} \subset\{r=1\}$, where $\left|c_{n}-b_{n}\right|<1 / 2^{n}$ and $\left|k_{n}-e_{n}\right|<1 / 2^{n}$, such that

$$
P_{n} \cap A^{-}=(-\infty, 0) \times\left[\pi_{\theta} b_{n}, \pi_{\theta} c_{n}\right]
$$

and

$$
Q_{n} \cap A^{+}=(1, \infty) \times\left[\pi_{\theta} e_{n}, \pi_{\theta} k_{n}\right] .
$$




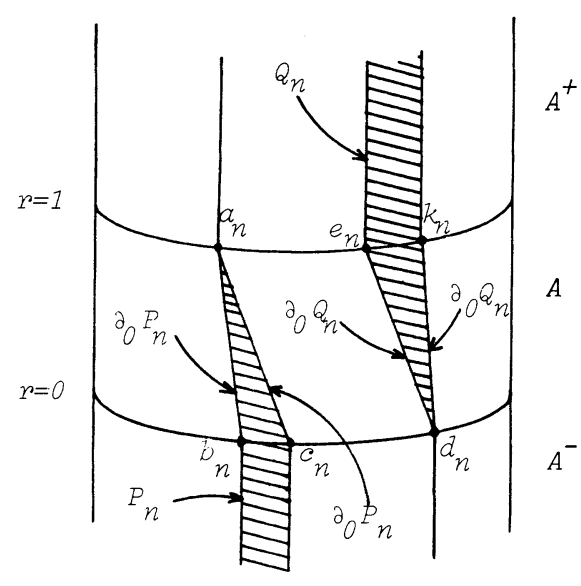

Figure 5a

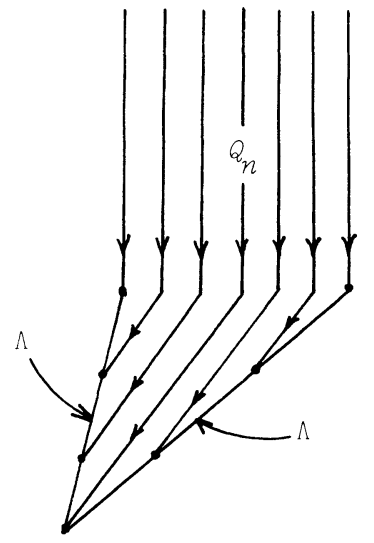

FIGURE $5 b$

Let $\partial_{0} P_{n}=\overline{a_{n} b_{n}} \cup \overline{a_{n} c_{n}}$ and $\partial_{0} Q_{n}=\overline{d_{n} e_{n}} \cup \overline{d_{n} k_{n}}$. Then

$$
\Lambda=\mathrm{Cl} \bigcup_{n \in \mathbf{Z}}\left[\partial_{0} P_{n} \cup \partial_{0} Q_{n}\right]
$$

Now let $\eta: \mathbf{R} \times S^{1} \supset$ be a continuous projection which collapses $\left\{P_{n}\right\}$ and $\left\{Q_{n}\right\}$ to the rays they replaced, preserves radial coordinates, and is a homeomorphism off $\bigcup_{n \in \mathbf{Z}}\left[P_{n} \cup Q_{n}\right]$. The desired homeomorphism $F: \mathbf{R} \times S^{1} \supset$ is the identity near $\{ \pm \infty\} \times S^{1}$ and can be written as $F=G \circ H$, where $H$ preserves radial coordinates and $\eta \circ H=f \circ \eta ; G$ commutes with $H$, preserves rays off $A$, is the identity on $\Lambda$, and makes $\Lambda$ an attracting set under $F$. One such $G$ preserves the piecewise linear foliation of $Q_{n}$, depicted in Figure 5b. As $n \rightarrow \infty$, the $C^{0}$ size of $\left[\left.G\right|_{P_{n}}-\right.$ Id] and $\left[\left.G\right|_{Q_{n}}-\right.$ Id $]$ goes to zero for all $n$.

Points on $\Lambda$, except those with radial coordinates equal to 0 or 1 , have dense orbits under $F$ on $\Lambda$ inherited from $f$. The two induced maps on prime ends are Denjoy. Briefly, since there are $C^{1}$ Denjoy maps on $S^{1}$ [Den], $F$ may be $C^{1}$-smoothed to a $C^{1}$ - diffeomorphism.

Example E. In Example $C$ we constructed the " $\sin 1 / x$ cobasin boundary" $\Lambda$ by irrational rotating and radially summing infinitely many rescaled Warsaw circles. One may instead irrationally rotate and radially sum double Warsaw circles where the two limit bars lie on disjoint orbits. After extension onto the plane by $F$, this "double $\sin (1 / x)$ cobasin" $\Lambda$ is minimal and induces irrational rotation on both circles of prime ends. As in Example D, wedge open one limit bar orbit from "outside" $\Lambda$, the other limit bar orbit from "inside" $\Lambda$. Compose with a homeomorphism under which points drift towards $\Lambda$ and which commutes with the Denjoy rotation. The new cobasin boundary is also minimal but the two maps on prime ends are Denjoy-like, thus not minimal.

\section{Example A}

The idea of this construction is to attach positive length "hairs" to one side of a circle forming a basin separating the invariant set $\Lambda_{0}$ in such a way that 


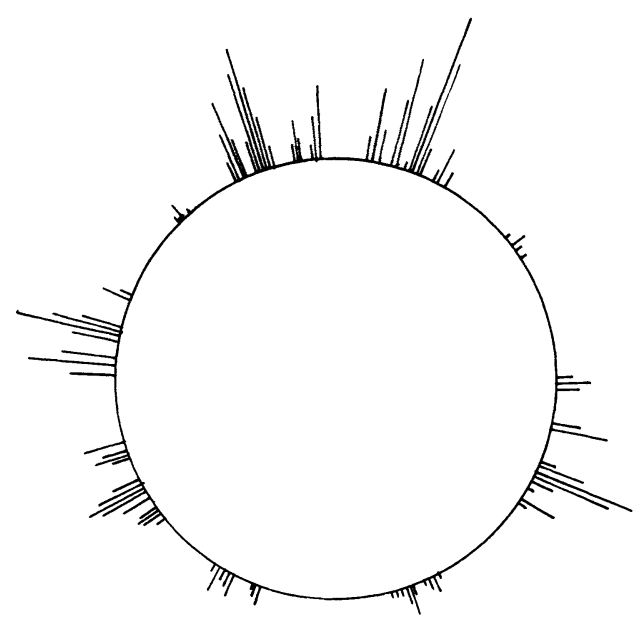

FigURE 6a. $\Lambda_{0}$

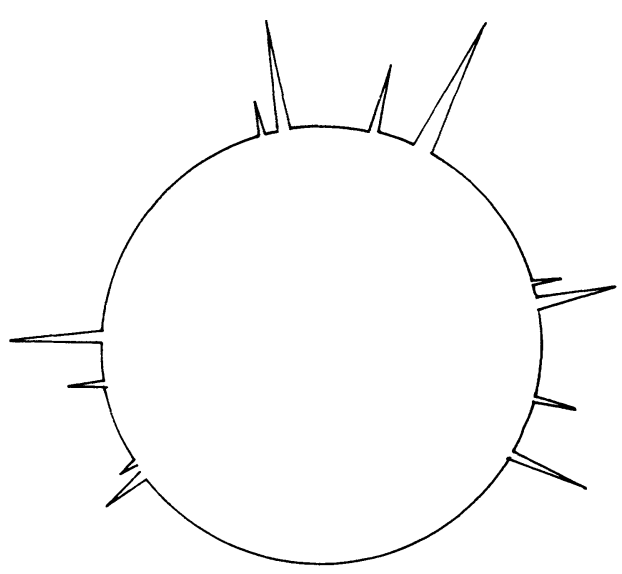

Figure $6 \mathrm{~b} . \Lambda$

the endpoints of the hairs are dense in $\Lambda_{0} . \Lambda_{0}$ is carried onto itself by a homeomorphism of the plane which is irrational rotation on rays. To complete the construction, a countable number of hairs of $\Lambda_{0}$ are wedged open from the inside forming the cobasin boundary $\Lambda$ (see Figure 6).

Again fix $\omega / 2 \pi \notin \mathbf{Q}$. Let $f_{w}: S^{1} \supset$ be rotation by $\omega$. Let $C$ be a Cantor set on $S^{1}$ with the property that $f_{\omega}^{n} C \cap C=\varnothing$ for all $n \neq 0$ (see M. Rees [R]). Let $\Sigma=\left\{\left(a_{1}, a_{2}, \ldots\right) \mid a_{i} \in\{0,1\}\right\}$ with the product topology and let $\Phi: \Sigma \rightarrow C$ be a homeomorphism such that $\Phi(\underline{a})$ is an endpoint of $C$ (accessible from $S^{1} \backslash C$ ) if and only if $\underline{a}$ ends in all 1's. Given $x \in C$, let $\underline{a}(x)=\left(a_{1}(x), a_{2}(x), \ldots\right)=\Phi^{-1}(x)$. Let $b_{i}(x)=a_{i}(x) / i$. Define $l_{0}: C \rightarrow[0, \infty)$ by

$$
l_{0}(x)=\prod_{i=1}^{\infty}\left(\frac{1}{2}\right)^{b_{i}(x)} .
$$

Extend $l_{0}$ onto $S^{1}$ by $l_{0}(x)=0$ if $x \notin C$. Next let $l: S^{1} \rightarrow[0, \infty)$ be given by

$$
l(x)=\sup _{n \in \mathbf{Z}} \frac{l_{0}\left(f_{\omega}^{n}(x)\right)}{2^{|n|}} .
$$

Let $\Lambda_{0}=\{(r, \theta) \mid 1 \leq r \leq 1+l(1, \theta)\}$.

The map $l$ is upper semicontinuous, thus $\Lambda_{0}$ is compact. $\mathbf{R} \backslash \Lambda_{0}$ is the union of two components $U_{1}$ and $U_{2}$ where $U_{1}$ is chosen bounded. $\partial U_{1}=\{r=1\}$ and $\partial U_{2}=\Lambda_{0}$ since $\left\{x \in S^{1} \mid l(x)=0\right\}$ is dense in $S^{1}$. The sides of the hairs $\Lambda_{0} \backslash\{r=1\}$ are covered up in the sense that if $x \in S^{1}$, there exists $\left\{x_{n} \rightarrow x\right\}$ and $\left\{y_{n} \rightarrow x\right\}$ with $x_{n}<x<y_{n}$, all $n$, such that $l\left(x_{n}\right) \rightarrow l(x)$ and $l\left(y_{n}\right) \rightarrow l(x)$. Thus any point of $\Lambda_{0}$ accessible from $U_{2}$ is an endpoint of a hair. 
Now define $F_{0}: \Lambda_{0} \rightarrow \Lambda_{0}$ by

$$
F_{0}(r, \theta)= \begin{cases}(1, \theta+\omega(\bmod 2 \pi)), & r=1, \\ (1 / 2 r+1 / 2, \theta+\omega(\bmod 2 \pi), & (1, \theta) \in \bigcup_{n=0}^{\infty} f_{\omega}^{n}(C), \\ (2 r-1, \theta+\omega(\bmod 2 \pi)), & (1, \theta) \in \bigcup_{n=1}^{\infty} f_{\omega}^{-n}(C),\end{cases}
$$

Then $F_{0}$ is a homeomorphism and $F_{0}\left(\Lambda_{0}\right)=\Lambda_{0} . \quad F_{0}$ extends to $U_{1}$ as $F_{0}(r, \theta)=(r, \theta+\omega(\bmod 2 \pi))$. An argument similar to those used in Example $\mathrm{C}$, shows that $F_{0}$ extends to $U_{2}$. Since every crosscut in $U_{2}$ nearly connects endpoints of hairs and $F_{0}$ rotates the endpoints by irrational rotation, every prime end under the induced homeomorphism on $\widehat{U}_{2}$ is nonwandering. Thus both induced homeomorphisms are conjugate to $f_{\omega}$.

Now wedge open $\Lambda_{0}$ from $U_{1}$ as in the construction of Example D. For fixed $\left(\alpha_{1}, \alpha_{2}, \ldots, \alpha_{k}\right) \in\{0,1\}^{\mathbf{N}}$ the set $\left\{\Phi(\underline{a}) \mid a_{i}=\alpha_{i}, i=1,2, \ldots, k\right\}$ is called a " $k$-block" of $C$. Over each $k$-block, for all $k \in \mathbf{N}$, choose a longest hair. Call the union of these hairs $A$ and let $\Lambda_{1}=\bigcup_{n \in Z} F_{0}^{n}(A)$. So $\Lambda_{1}$ is a countable union of hairs, dense in $\Lambda_{0}$. Wedged open the rays through $\Lambda_{1}$ from $U_{1}$, as in Example D, forming $\Lambda$, the closure of the wedge open hairs of $\Lambda_{1}$.

Carry wedge to wedge via a planar homeomorphism $H$ which covers $F_{0}$. Our homeomorphism $F=G \circ H$, where $G$ commutes with $H$ and makes $\Lambda$ attracting, as in Example D.

The induced map on prime ends from the unbounded component of $\mathbf{R}^{2} \backslash \Lambda$ remains conjugate to that of $\widehat{U}_{2}$ induced by $F_{0}$; it is irrational rotation. From the bounded component, the induced homeomorphism on prime ends is "countably Denjoy-like." Such circle homeomorphisms are constructed from irrational rotations by the removal of a countable number of pairwise disjoint orbits. These orbits are replaced by a countable number of closed intervals with finite total length. All Denjoy-like homeomorphisms can be made $C^{1}$. Thus $F$ may be $C^{1}$-smoothed.

Acknowledgments. The author would like to thank Marcy Barge and Richard Gillette for their technical assistance in the construction of several of the examples communicated in this manuscript.

\section{REFERENCES}

[AY] K. Alligood and J. Yorke, Accessible saddles on fractal basin boundaries, preprint, 1988.

[AS] K. Alligood and T. Sauer, Rotation numbers of periodic orbits in the Hénon map, preprint 1988.

[BG] M. Barge and R. Gillette, Indecomposability and dynamics of invariant plane separating continua, preprint 1988.

[Be1] A. S. Besicovitch, A problem on topological transformations of the plane, Fund. Math. 28 (1937).

[Be2] _ A problem on topological transformations of the plane. II, Cambridge Philos. Soc. Proc. Math. 47 (1951).

[Bi] G. D. Birkhoff, Sur quelques courbes fermées remarquables, Bull. Soc. Math. France 60 (1932). 
[C] C. Carathéodory, Uber die Begrenzung einfach zusammenhangender Gebiete, Math. Ann. 73 (1913).

[CL1] M. L. Cartwright and J. E. Littlewood, Some fixed point theorems, Ann. of Math. (2) 54 (1951), 1-37.

[CL2] _ On non-linear differential equations of the second order..., J. London Math. Soc. 20 (1945), 180-189.

[Den] A. Denjoy, Sur les courbes definies par les equations differentielles a la surface de tore, J. Math. Pures Appl. 11 (1932), 333-375.

[Dev] R. Devaney, An introduction to chaotic dynamical systems, Benjamin-Cummings, Menlo Park, Calif., 1986.

[GH] W. H. Gottschalk and G. A. Hedlund, Topological dynamics, Amer. Math. Soc. Colloq. Publ., vol. 36, Amer. Math. Soc., Providence, R. I., 1955, pp. 139-142.

$[\mathrm{H}] \quad$ M. Handel, A pathological area preserving $C^{\infty}$ diffeomorphism of the plane, Proc. Amer. Math. Soc. 86 (1982).

[K] K. Kuratowski, Topology, vol. II, Academic Press, New York, 1968.

[L] M. Levi, Qualitative analysis of the periodically forced relaxation oscillations, Mem. Amer. Math. Soc. No. 244 (1981).

[M] J. Mather, Topological proofs of some purely topological consequences of Carathéodory's theory of prime ends, Selected Studies (Th. M. Rassias and G. M. Rassias, eds.), NorthHolland, 1982, pp. 225-255.

[Ne] M. Newman, Elements of the topology of plane sets of points, 2nd ed., Cambridge Univ. Press, 1954.

[Ni] Z. Nitecki, Differentiable dynamics, an introduction to the orbit structure of diffeomorphisms, The M.I.T. Press, Cambridge, Mass., 1971.

[R] M. Rees, A minmal positive entropy homeomorphism of the 2-torus, J. London Math. Soc. (2) 23 (1981), 537-550.

National Science Foundation, 1800 G Street, Washington, D.C. 20550

Department of Mathematics, Montana State University, Bozeman, Montana 59717 